\title{
MicroRNA-6852 suppresses glioma A172 cell proliferation and invasion by targeting LEF1
}

\author{
JIALIANG WANG, HAIPENG LIU, KEBIN ZHENG, SHUAI ZHANG and WEI DONG \\ Department of Neurosurgery, Affiliated Hospital of Hebei University, Baoding, Hebei 071000, P.R. China
}

Received November 22, 2018; Accepted May 23, 2019

DOI: 10.3892/etm.2019.7762

\begin{abstract}
RNA (miR)-6852 has been demonstrated to suppress the progression of gastric, colorectal and cervical cancer. The mechanism by which miR-6852 regulates glioma cells is yet to be elucidated. In the present study, reverse transcription-quantitative PCR analysis was used and the results demonstrated that miR-6852 expression was reduced in glioma tissues and cells. Cell counting kit- 8 and transwell assay analysis indicated that proliferation, migration and invasion of A172 cells in the miR-6852 mimic group were lower than in the miR-NC group. Compared with the Inh-NC group, A172 cells of the Inh-miR-6852 group exhibited higher proliferation, migration and invasion. Additionally, the results indicated that lymphoid enhancer binding factor 1 (LEF1) was directly inhibited by miR-6852 and LEF1 expression was negatively correlated with miR-6852 expression in glioma tissues. Furthermore, the restoration of LEF1 reversed the effects of the miR-6852 mimics. The present findings suggested that miR-6852 inhibited glioma cells proliferation, migration and invasion by targeting the suppression of LEF1.
\end{abstract}

\section{Introduction}

Glioma is the most common type of central nervous system tumor in the clinical setting (1). Gliomas exhibiting a high pathological grade indicate strong invasiveness. In most cases, the boundaries between tumor and normal tissue are blurred and in high-grade patients, can result in the incomplete surgical removal of diseased tissues (1). In addition, high-grade glioma is more resistant to radiation and chemotherapy $(2,3)$.

microRNAs (miRs or miRNAs) are a class of endogenous non-coding small RNAs, 18-24 nucleotides in length, that are ubiquitous in viruses and eukaryote cells in highly conserved sequences. It has been estimated that up to $50 \%$ of human

Correspondence to: Dr Wei Dong, Department of Neurosurgery, Affiliated Hospital of Hebei University, 212 Yuhua East Road, Baoding, Hebei 071000, P.R. China

E-mail: weidong08@163.com

Key words: glioma, microRNA-6852, lymphoid enhancer binding factor 1 , proliferation, migration, invasion protein-coding genes are regulated by miRNAs (4). Each miRNA has the potential to regulate hundreds of RNAs, and their expression is associated with tissue status and disease subtype (5). miRNAs have been demonstrated to serve an important role in the development and prognosis of glioma. Lv et al (4) reported that low miR-320b expression in glioma was associated with poor patient prognosis. The upregulation of miR-320b enhanced apoptosis and attenuated the proliferation, migration and invasive abilities of glioma cells. Santangelo et al (6) collected serum samples from patients with glioma and observed an increased expression of miR-21, miR-222 and miR-124-3p in the serum exosomes of these patients when compared with healthy participants. Patients with high-grade glioma exhibited increased miR-21, miR-222 and miR-124-3p expression when compared with the other patients with low-grade glioma. Additionally, miR-211 was also indicated to be reduced in glioma, and was closely associated with a decreased patient survival time and considered to be an independent risk factor for glioma prognosis (7). For high-grade glioma, the upregulation of miR-126 may impair the invasive ability of glioma cells by targeting KRAS, meaning miR-126 could be an effective therapeutic target (8). Therefore, these genes are considered to be a potential biological target for glioma prognosis and treatment.

Recently, miR-6852 has been demonstrated to be associated with the development of tumors, including those in gastric, colorectal and cervical cancer (9-11). The association of miR-6852 with glioma remains undetermined. Therefore, the present study aimed to assess miR-6852 expression in glioma, and further determine the effect of miR-6852 in the regulation of glioma progression.

\section{Materials and methods}

Glioma tissue collection. A total of 32 pairs of glioma tissues (14 males and 18 females; age range, 31-56 years old; median age, 47 years old) and corresponding normal tissues were collected during biopsy from Affiliated Hospital of Hebei University (Hebei, China) between October 2014 and October 2016 for the current study. Normal tissues were derived from the temporal lobes and saddle area $2 \mathrm{~cm}$ away from tumor tissues. All glioma tissues were obtained from patients upon first diagnosis of glioma. Among these patients, 18 cases were high-grade and 14 cases were low-grade. All patients did not recieve radiotherapy or chemotherapy prior to surgery. Patients 
treated by radiotherapy or chemotherapy prior to surgery were excluded. All participates had signed informed consent and the present study has been approved by The Ethics Committee of Affiliated Hospital of Hebei University.

Cell culture. Normal human astrocytes (NHAs; ScienCell Research Laboratories, Inc.) were maintained in astrocyte medium (ScienCell Research Laboratories, Inc.) according to manufacturers protocol. In addition, human glioma cell lines (U251 and A172 cells), purchased from the Type Culture Collection of the Chinese Academy of Sciences were incubated at $37^{\circ} \mathrm{C}, 5 \% \mathrm{CO}_{2}$ in DMEM (Gibco; Thermo Fisher Scientific, Inc.) containing 10\% FBS (Gibco; Thermo Fisher Scientific, Inc.), $100 \mathrm{mg} / \mathrm{ml}$ streptomycin and $100 \mathrm{U} / \mathrm{ml}$ penicillin (Gibco; Thermo Fisher Scientific, Inc.).

Cell transfection. The mature miR-6852 mimics (5'-CCC UGGGGUUCUGAGGACAUG-3'), miR-6852 inhibitors (5'-CATGTCCTCAGA ACCCCAGGG-3') and negative control (NC; 5'-UCACAACCUCCUAGAAAGAGUAGA-3') were synthesized and verified by Shanghai GenePharma Co., Ltd. The coding sequence of lymphoid enhancer binding factor 1 (LEF1) was constructed into the pcDNA3 vector (Invitrogen; Thermo Fisher Scientific, Inc.) to generate a pcDNA3-LEF vector for the overexpression of LEF1. The pcDNA3 empty vector was used for overexpressing control. A172 human glioma cells were cultured under the aforementioned standard culture conditions and prepared as a single cell suspension $\left(1 \times 10^{6}\right.$ cells $\left./ \mathrm{ml}\right)$ with serum free DMEM. Cells were subsequently seeded into six-well plates with $1 \mathrm{ml}$ of cell suspension per well. A period of 1 day later, cells were grouped and transfected as follows: miR-NC group (50 nM), cells were transfected with a miR-6852 mimics negative control (50 nM); miR-6852 group (50 nM), cells were transfected with miR-6852 mimics (50 nM); Inh-NC (5'-GCG UAACUAAUACAUCGGAUUCGU-3') group (50 nM), cells were transfected with a miR-6852 inhibitor negative control; Inh-miR-6852 group, cells were transfected with miR-6852 inhibitors; and miR-6852 + pcDNA3-LEF1 group, cells were co-transfected with miR-6852 mimics and LEF1 overexpression vectors. Transfection was performed using Lipofectamine $2000^{\mathrm{Tм}}$ (Thermo Fisher Scientific, Inc.), according to manufacturer's protocol. After $6 \mathrm{~h}$, successfully transfected cells were confirmed by qRT-PCR analysis and cultured in six-well plates using DMEM containing $10 \%$ FBS for 2 days at $37^{\circ} \mathrm{C}$ and $5 \% \mathrm{CO}_{2}$.

Cell Counting Kit (CCK)- 8 assay. A total of $1 \times 10^{4}$ A172 cells of each aforementioned group were inoculated in 96-well plates with $100 \mu \mathrm{l}$ DMEM, containing $10 \% \mathrm{FBS}$, and cultured at $37^{\circ} \mathrm{C}$ in $5 \% \mathrm{CO}_{2}$ for $0,24,48$ or $72 \mathrm{~h}$. At each time point, cells were removed from the incubator, and $10 \mu \mathrm{l} \mathrm{CCK}-8$ solution (Beyotime Institute of Biotechnology) was added into each well for subsequent incubation for $4 \mathrm{~h}$ at $37^{\circ} \mathrm{C}$. The optical density (OD) of each well was monitored using an enzyme immunoassay analyzer (INC-STAR Corp.) at $450 \mathrm{~nm}$.

Transwell experiments. A period of 2 days after transfection, A172 cells of each aforementioned group were prepared as single cell suspensions $\left(1 \times 10^{5}\right.$ cells $\left./ \mathrm{ml}\right)$ with serum-free
DMEM. Transwell chambers were inserted into 24-well plates containing $600 \mu \mathrm{l}$ of DMEM (with 10\% FBS) in the lower chamber. On the upper chamber, $100 \mu 1$ of cell suspension was added with serum-free DMEM. All plates were cultured for $48 \mathrm{~h}$ at $37^{\circ} \mathrm{C}, 5 \% \mathrm{CO}_{2}$ in the incubator. Non-migrated cells were scraped off and migrated cells were fixed using $4 \%$ formaldehyde for $30 \mathrm{~min}$ at room temperature, stained using $0.5 \%$ crystal violet for $30 \mathrm{~min}$ at room temperature and counted under a light microscope (magnification, x200). The aforementioned procedure was also used to detect cell invasion except Matrigel (BD Biosciences) was spread onto the Transwell chamber.

Luciferase reporter gene assay. LEF1 was predicted as a potential miR-6852 target using TargetScan7 (http://www.targetscan. org/vert_71/). The LEF1 3'-UTR region was constructed into the pGL3 luciferase reporter vector (Promega Corporation) to generate pGL3-LEF1-wild-type (WT) reporter. The binding site for miR-6852 in LEF1 3'-UTR was mutated to obtain the pGL3-LEF1-mutant reporter. For the luciferase reporter assay, A172 cells in the miR-NC and miR-6852 group underwent subsequent transfection with pGL3-LEF1-WT or pGL3-LEF1-mutant respectively using Lipofectamine $2000^{\mathrm{TM}}$. Cells were incubated at $37^{\circ} \mathrm{C}, 5 \% \mathrm{CO}_{2}$ for 1 day. Luciferase activity of each well was measured using a Dual-Luciferase Reporter System (Promega Corporation) and normalized to Renilla luciferase activity.

$R N A$ extraction and reverse transcription-quantitative (RT-q) $P C R$. Total RNA was extracted from glioma, normal tissues and glioma cell lines using TRIzol reagent (Thermo Fisher Scientific, Inc.). cDNA template synthesis was performed using the Prime Script RT Reagent kit (Takara Bio, Inc.) according to the manufacturer's protocol. The reaction was performed with incubation at $42^{\circ} \mathrm{C}$ for $1 \mathrm{~h}$, and the enzyme was subsequently inactivated by incubation at $85^{\circ} \mathrm{C}$ for $5 \mathrm{~min}$.. miR-6852 expression was determined using SYBR Premix Ex Taq II (GeneCopoeia, Inc.) according to the following conditions: $10 \mathrm{~min}$ of pre-denaturation at $95^{\circ} \mathrm{C}$, followed by 40 cycles of $10 \mathrm{sec}$ denaturation at $95^{\circ} \mathrm{C}, 20 \mathrm{sec}$ annealing at $60^{\circ} \mathrm{C}$ and $30 \mathrm{sec}$ extension at $72^{\circ} \mathrm{C}$. LEF1 mRNA expression was measured using a SYBR Green PCR Master Mix (Applied Biosystems; Thermo Fisher Scientific, Inc.) with the following procedures: $10 \mathrm{~min}$ pre-denaturation at $95^{\circ} \mathrm{C}$, followed by 36 cycles of $10 \mathrm{sec}$ denaturation at $95^{\circ} \mathrm{C}, 20 \mathrm{sec}$ annealing at $60^{\circ} \mathrm{C}$ and $34 \mathrm{sec}$ extension at $72^{\circ} \mathrm{C}$. U6 and GAPDH were used as internal references for miR-6852 and LEF1 mRNA expression, respectively. Relative miR-6852 and LEF1 mRNA were processed using the $2{ }^{\Delta \Delta \mathrm{Cq}}$ method (12). The primer sequences were: miR-6852 forward, 5'-AACGAGACGACGACAGAC-3' and reverse, 5'-CCCTGGGGTTCTGAGGACATG-3'; U6 forward, 5'-GTGCTCGCTTCGGCAGCACAT-3' and reverse, 5'-TACCTTGCGAAGTGCTTAAAC-3'; LEF1 forward, 5'-AGAACACCCCGATGACGGA-3' and reverse, 5'-GAG GGTCCCTTGTTGTAGAGG-3'; and GAPDH forward, 5'-ACCCAGAAGACTGTGGATGG-3' and reverse, 5'-TCT AGACGGCAGGTCAGGTC-3'.

Western blot analysis. Total proteins in each cell sample were extracted using RIPA lysis buffer (Beyotime Institute of 

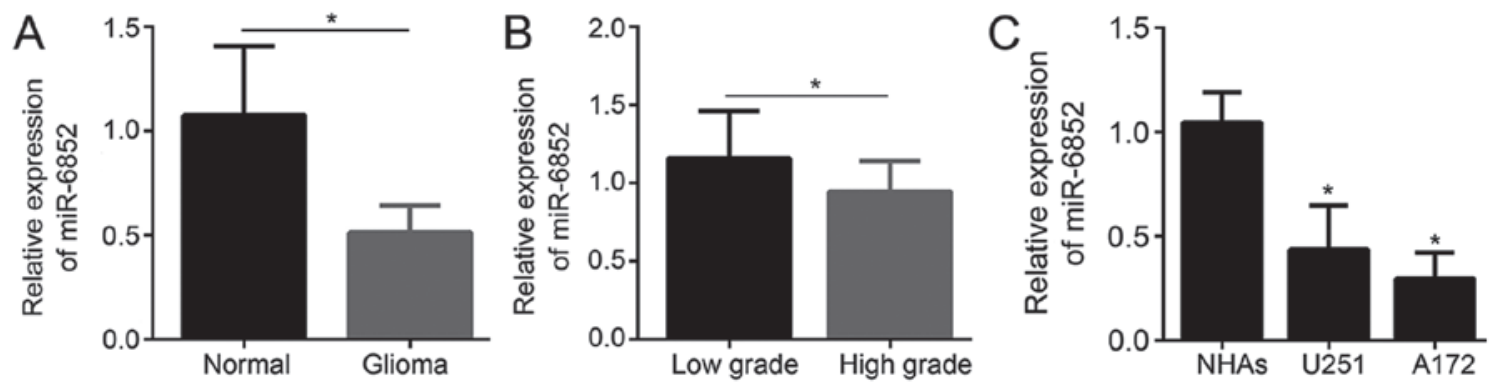

Figure 1. miR-6852 expression is reduced in glioma tissues and cells. RT-qPCR analysis of miR-6852 expression in (A) glioma tissues and corresponding normal tissues and (B) low-grade and high-grade glioma tissues. "P<0.05 as indicated. RT-qPCR analysis of miR-6852 expression in (C) NHA, U251 and A172 cells. "P<0.05 vs. NHAs. miR, microRNA; RT-q, reverse transcription-quantitative; NHAs, normal human astrocytes.

Biotechnology). Proteins were then quantified using a bicinchoninic acid kit (Pierce; Thermo Fisher Scientific, Inc.), then $40 \mu \mathrm{g}$ protein/lane were separated on $10 \%$ SDS-PAGE, then transferred to a polyvinylidene difluoride membrane (PVDF) for $2 \mathrm{~h}$. The PVDF membrane was subsequently incubated with $5 \%$ skim milk for $1 \mathrm{~h}$ at room temperature. Tris buffered saline with Tween 20 (TBST) was used to wash the membrane. LEF1 primary antibody (1:1,000; cat. no. ab137872, Abcam) was used to incubate the membrane for $12 \mathrm{~h}$ at $4^{\circ} \mathrm{C}$. Horseradish peroxidase-conjugated secondary antibodies (1:5,000; cat. no. ab7090; Abcam) was then used to further incubate the membrane at room temperature for $2 \mathrm{~h}$. The membrane was washed with TBST 3 times for 15 min each time. LEF1 protein expression was monitored using the Pierce ${ }^{\mathrm{TM}}$ ECL Western Blotting Substrate (Thermo Fisher Scientific, Inc.) and quantified using Quantity One software v4.62 (Bio-Rad Laboratories Inc.). GAPDH was used as the internal reference.

Statistical analysis. All data included in the current study was repeated three times and is expressed as the mean \pm standard deviation. A Student's t-test was used to analyze the differences between two groups. A one-way ANOVA followed by a Tukey's post-hoc test was used for multiple comparisons. A Pearson's correlation coefficient analysis was used to determine the correlation between miR-6852 and LEF1 expression. SPSS 19.0 (IBM Corp.) was used for statistical analysis. $\mathrm{P}<0.05$ was considered to indicate a statistically significant difference.

\section{Results}

miR-6852 expression is reduced in glioma tissues and cells. Previous reports have indicated the tumor-suppressive roles of miR-6852 in a variety of cancer types, including gastric and colorectal cancer (9-11). Therefore, it is reasonable to speculate that miR-6852 may be associated with glioma. miR-6852 expression was assessed using RT-qPCR in 32 pairs of glioma and corresponding normal tissue samples. As demonstrated in Fig. 1A, when compared with normal tissues, glioma tissues exhibited significantly decreased relative miR-6852 expression $(\mathrm{P}<0.05)$. Tissues of high-grade glioma $(\mathrm{n}=18)$ exhibited significantly decreased relative miR-6852 expression when compared with tissues of low-grade glioma $(\mathrm{n}=14 ; \mathrm{P}<0.05$; Fig. 1B). miR-6852 expression was significantly decreased in glioma cells (including U251 and A172 cells) when compared with expression in NHA cells $(\mathrm{P}<0.05$; Fig. $1 \mathrm{C})$.
miR-6852 upregulation inhibits A172 cell proliferation, migration and invasion. A172 miR-6852 expression significantly increased subsequent to transfection with miR-6852 mimics $(\mathrm{P}<0.05$; Fig. 2A). A period of $72 \mathrm{~h}$ after transfection, A172 cells in the miR-6852 group revealed a significantly decreased OD450 value when compared with the miR-NC group $(\mathrm{P}<0.05$; Fig. 2B). The results of the transwell assay revealed that A172 cells in the miR-6852 group also exhibited a decreased number of migrated and invasive cells when compared with those in the miR-NC group (all, $\mathrm{P}<0.05$; Fig. 2C and D).

miR-6852 downregulation promotes A172 cell proliferation, migration and invasion. A172 cells transfected with miR-6852 inhibitors and miR-6852 inhibitor negative controls. As presented in Fig. 3A, transfection with the Inh-miR-6852 inhibitor decreased relative miR-6852 expression in A172 cells $(\mathrm{P}<0.05)$. This decreased miR-6852 expression significantly enhanced A172 cell proliferation at $72 \mathrm{~h}$ subsequent to transfection, as indicated by the higher OD450 value of A172 cells in the Inh-miR-6852 group compared with the Inh-NC group $(\mathrm{P}<0.05$; Fig. 3B). In addition, the number of migrated and invasive cells of the Inh-miR-6852 group was significantly increased when compared with the number exhibited in the Inh-NC group ( $\mathrm{P}<0.05$; Fig. $3 \mathrm{C}$ and $\mathrm{D})$.

LEF1 expression is inhibited by miR-6852. TargetScan predicted that miR-6852 has a direct binding site with LEF1. Previous studies have also indicated that miR-6852 targets forkhead box protein J1 (FOXJ1) and transcription factor 7 (TCF7) in a variety of other types of cancer $(9,10)$. Therefore, in the present study, WT and mutant sequences of LEF1 were constructed (Fig. 4A). Furthermore, FOXJ1 and TCF7 containing the binding sites for miR-6852 were designed (data not shown). The luciferase reporter gene assay indicated that insertion of LEF1 mutant-type sequences did not influence the relative luciferase activity of A172 cells in the miR-NC and miR-6852 group. However, LEF1 wild-type sequence insertion significantly reduced the relative luciferase activity of A172 cells in the miR-6852 group when compared with the miR-NC group ( $\mathrm{P}<0.05$; Fig. 4B), indicating that LEF1 expression was directly suppressed by miR-6852. Furthermore, the FOXJ1 or TCF7 mutations did not influence the relative luciferase activity in A172 cells (data not shown), indicating that miR-6852 targets LEF1 in 
A

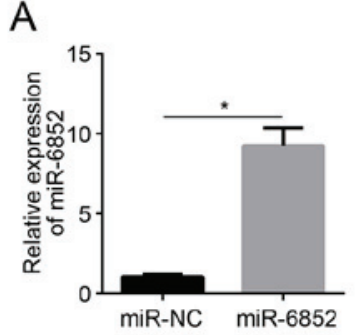

$\mathrm{B}$
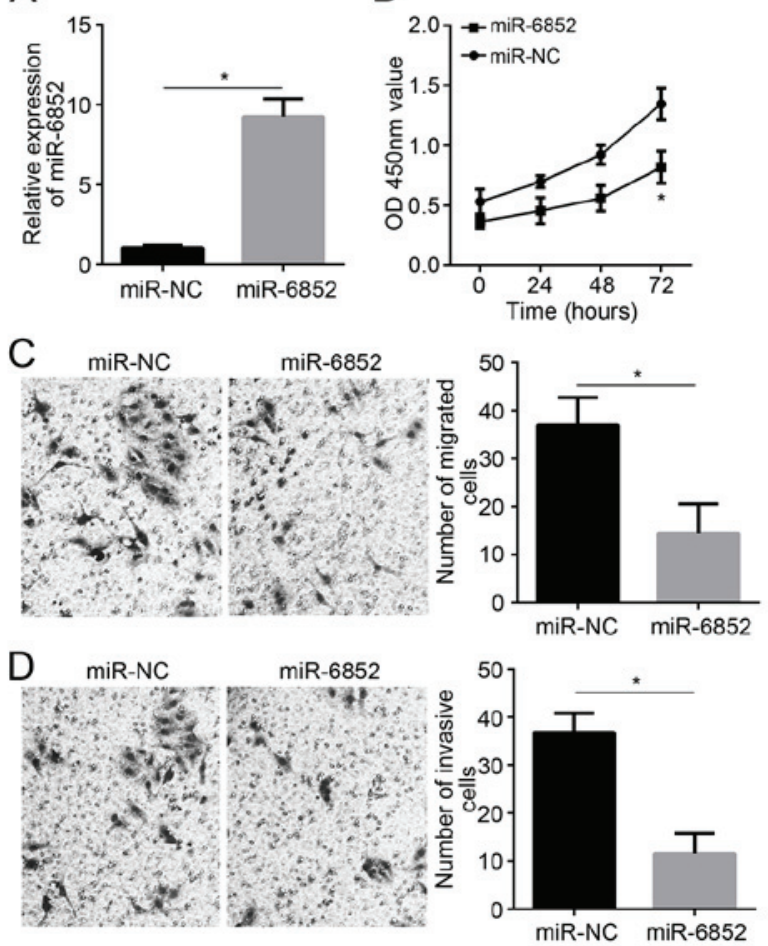

Figure 2. miR-6852 upregulation inhibits A172 cell proliferation, migration and invasion. (A) Reverse transcription-quantitative PCR and (B) cell counting kit-8 analysis of A172 cell miR-6852 expression in the miR-NC and miR-6852 group. Transwell assay analysis of A172 cell (C) migration and (D) invasion in the miR-NC and miR-6852 group (magnification, $\mathrm{x} 200$ ). ${ }^{*} \mathrm{P}<0.05$ vs. miR-NC group or as indicated. miR, microRNA; NC, negative control; OD, optical density.

glioma. In addition, A127 cell LEF1 mRNA expression in the miR-6852 group was significantly deceased compared with the expression in the miR-NC group $(\mathrm{P}<0.05$; Fig. $4 \mathrm{C})$. LEF1 protein level was also decreased by miR-6852 (Fig. 4D). Pearson's correlation analysis demonstrated that LEF1 expression was negatively correlated with miR-6852 expression in glioma tissues (Fig. 4E).

LEF1 overexpression reverses the impaired proliferation, migration and invasion of A172 cells induced by miR-6852. LEF1 protein expression in A172 cells was markedly upregulated after transfection with pcDNA3-LEF1 overexpression vectors (Fig. 5A). A172 cell proliferation, migration and invasion in the miR-NC + pcDNA3 vector group, miR-6852 + pcDNA3 vector group, miR-6852 + pcDNA3-LEF1 group and miR-NC + pcDNA3-LEF1 group were also assessed. At $72 \mathrm{~h}$, the OD450 value of A172 cells in the miR-6852 + pcDNA3 vector group was lower than that exhibited in the miR-NC + pcDNA3 vector group, miR-NC + pcDNA3-LEF1 group and miR-6852 + pcDNA3-LEF1 group $(\mathrm{P}<0.05$; Fig. 5B). In addition, the miR-6852 + pcDNA3 vector group exhibited a significant decline in the number of migrated and invasive cells when compared with those in the miR-6852+ pcDNA3-vector group $(\mathrm{P}<0.05$; Fig. $5 \mathrm{C}$ and $\mathrm{D})$. The results indicated that LEF1 overexpression reversed the impaired proliferation, migration and invasion of A172 cells induced by miR-6852.
A

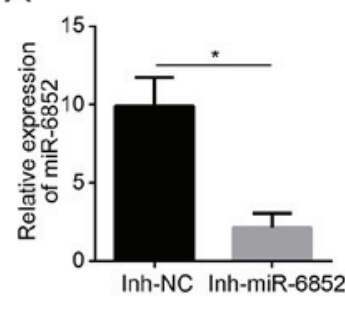

B
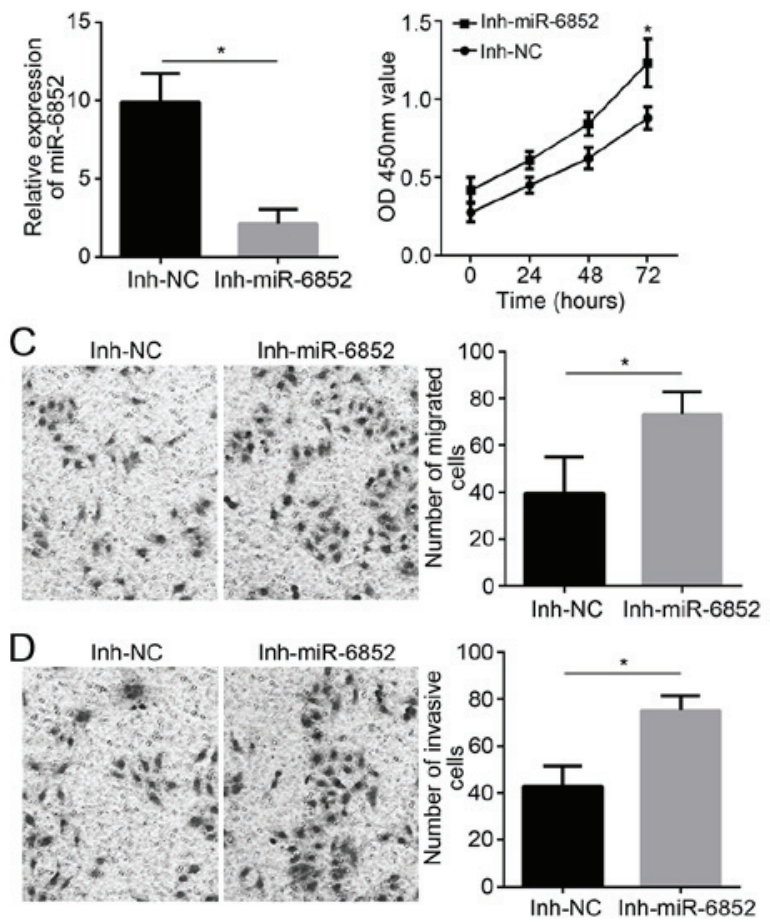

Figure 3. miR-6852 downregulation promotes A172 cell proliferation, migration and invasion. Reverse transcription-quantitative PCR analysis of (A) A172 cell miR-6852 expression of the Inh-NC and Inh-miR-6852 group. Cell counting kit- 8 assay of (B) A172 cells proliferation ability in the Inh-NC and Inh-miR-6852 group. Transwell assay analysis of A172 cell (C) migration and (D) invasion ability of the Inh-NC and Inh-miR-6852 group (magnification, $\mathrm{x} 200)$. ${ }^{*} \mathrm{P}<0.05$ vs. Inh-NC group or as indicated. miR, microRNA; inh, inhibitor; NC, negative control; OD, optical density.

\section{Discussion}

Glioma is one of the most deadly malignancies worldwide (1). Currently, the standard treatment for glioma is surgical resection and adjuvant chemotherapy and radiotherapy (1). However, due to treatment resistance and tumor recurrence, these treatment strategies do not achieve the desired therapeutic effect (13). The treatment of glioma is a clinical problem that requires urgent attention.

The understanding of the molecular pathology of glioma had led to the discovery of underlying mechanisms that are associated with glioma development and progression. Previous studies have demonstrated that in addition to a large number of coding genes, various non-coding genes (particularly miRNAs) are also associated with the regulation of glioma development $(14,15)$. A study of 148 clinical specimens revealed a significant decrease in miR-424 expression in glioma tumor tissues compared with the corresponding adjacent normal tissues (14). Results of an in vitro cell assay indicated the enhanced apoptotic ability and decreased invasive and migration abilities of glioma cells after miR-424 upregulation (14). Overexpressed miR-130b was demonstrated to suppress glioma cell apoptosis by selectively targeting CYLD, which is associated with poor prognosis of patients with glioma (15). Therefore, miR-130b was considered as an independent predictor of poor prognosis in patients with 
A

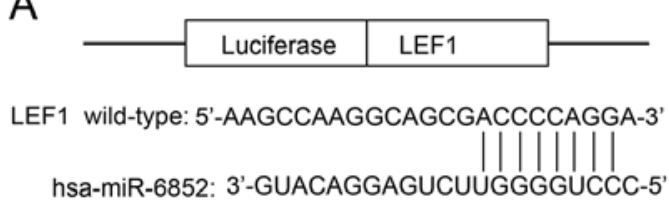

LEF1 mutant: 5'-AAGCCAAGGCAGCGUGGGGUCCA-3'

C
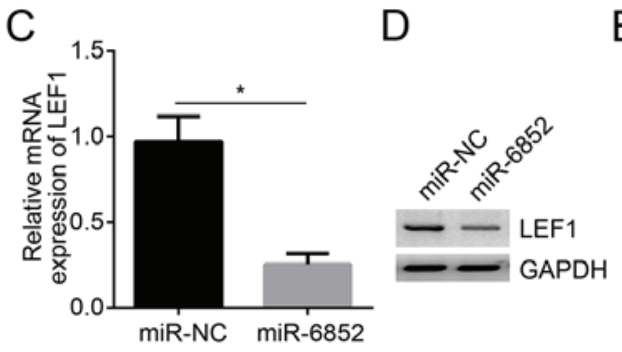

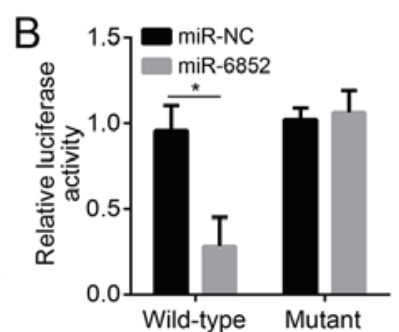

$\mathrm{E}$

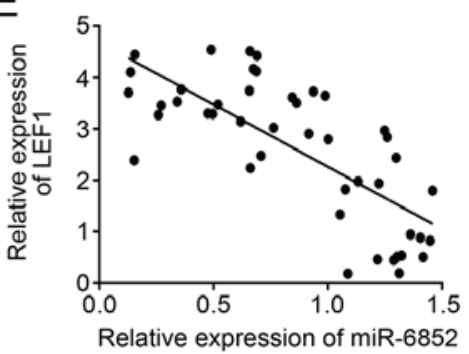

Figure 4. LEF1 is directly inhibited by miR-6852. (A) Wild-type and mutant-type sequences of LEF1 containing the binding site. (B) A luciferase reporter gene assay analysis of the relationship between miR-6852 and LEF1. (C) Reverse transcription-quantitative PCR analysis of LEF1 mRNA and (D) western blot analysis of LEF1 protein expression in A172 cells in the miR-6852 and miR-NC group. (E) Pearson correlation analysis of the correlation between miR-6852 and LEF1 expression in glioma tissues. " $\mathrm{P}<0.05$ as indicated. LEF1, lymphoid enhancer binding factor 1; miR, microRNA; NC, negative control.

A

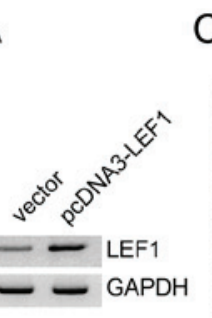

C
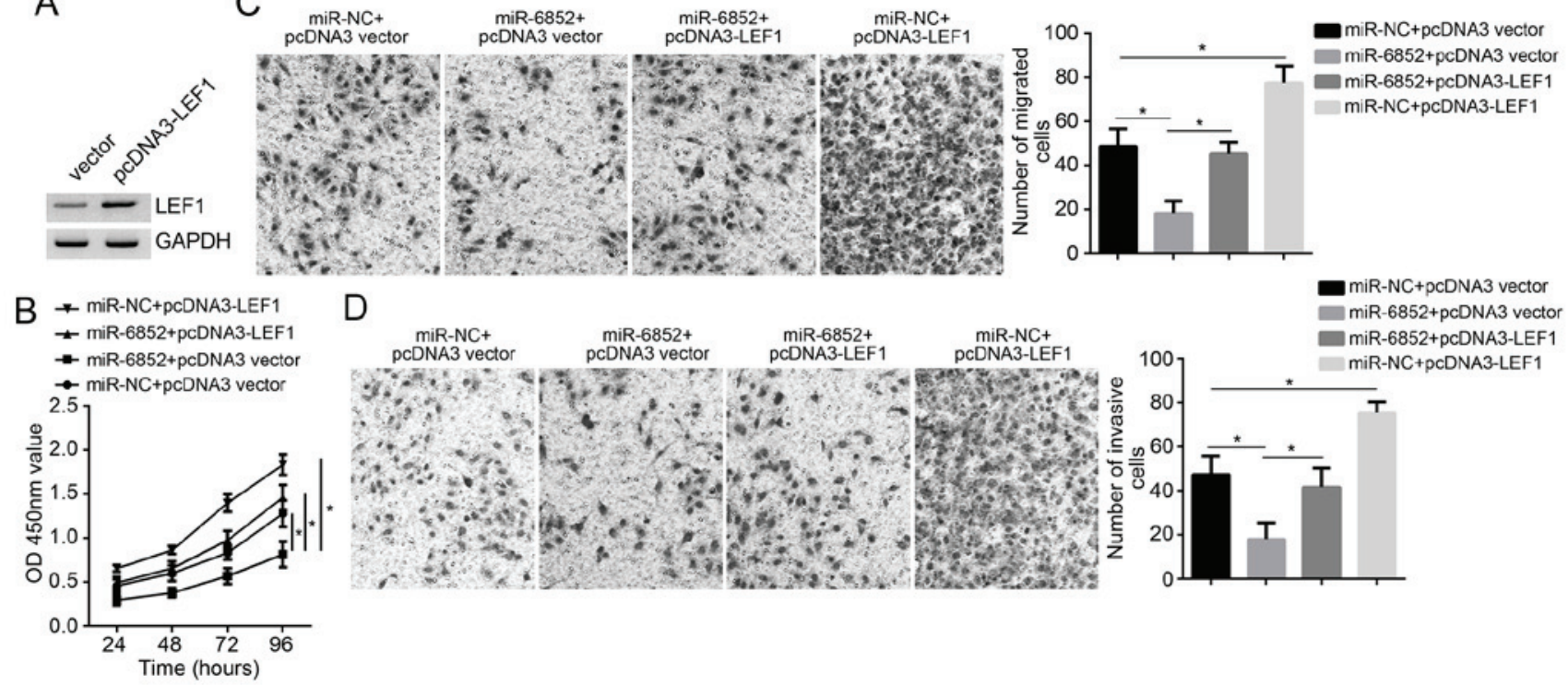

Figure 5. LEF1 overexpression reverses the impaired proliferation, migration and invasion of A172 cells induced by miR-6852. (A) Western blot analysis of LEF1 protein expression in A172 cells of the vector and pcDNA3-LEF1 group. (B) Cell counting kit-8 analysis of A172 cell proliferation. Transwell assay analysis of A172 cell (C) migration and (D) invasion. ${ }^{*} \mathrm{P}<0.05$ vs. miR-6852 + pcDNA3 vector group or as indicated. LEF1, lymphoid enhancer binding factor 1; miR, microRNA; NC, negative control.

glioma (15). In the current study, miR-6852 expression in glioma tissues and cells was decreased. High-grade glioma tissues exhibited lower miR-6852 expression than low-grade glioma tissues. Poudyal et al (11) revealed that the upregulation of miRNA-6852 could arrest cervical cancer cells at the $G_{2} / M$ phase and induce cell necrosis. Cui and Hong (9) demonstrated that miR-6852 expression was reduced in colorectal cancer and was closely associated with the metastasis and poor prognosis of patients. In vitro, colorectal cancer cell invasion and proliferation abilities and the proportion of cells in the $\mathrm{S}$ phase were significantly reduced after miR-6852 overexpression. This research also indicated that miR-6852 overexpression could suppress glioma cell proliferation, migration and invasion, while miR-6852 downregulation had the opposite effect.

LEF1 exerts an important regulatory role in the development of a variety of tissue types and its abnormal expression and dysfunction could lead to developmental disorders of tissues (16-18). LEF1 is highly expressed in numerous animal embryonic tissues and its expression rapidly decreases as the individual matures (16-18). However, previous research has demonstrated that LEF1 is upregulated in multiple tumor types. Fang et al (19) demonstrated that LEF1 is a novel oncogene that promoted hepatocellular carcinoma cell proliferation, invasion 
and migration abilities and self-renewal ability. Increased LEF1 expression was associated with advanced TNM stage, lymph node metastases and the reduced overall survival of patients with colorectal cancer (20). Gao et al (21) revealed that, in glioblastoma multiforme, LEF1 silencing suppressed U251 cell proliferation, invasion and migration, indicating that LEF1 could be considered a novel treatment target for glioblastoma multiforme. In glioma, elevated LEF-1 expression was one of the hallmarks of malignant glioma, and this could be used as an important marker for malignant transformation (22). It has been previously reported that LEF1 is associated with the signal transduction of $\mathrm{Wnt} / \beta$-catenin downstream molecules. Previous studies have revealed that LEF1 binds to $\beta$-catenin to form a $\beta$-catenin/LEF complex, thereby promoting the expression of Wnt signaling pathway downstream molecules, including c-Myc and Cyclin D1 $(23,24)$. LEF1 tumor expression was also mediated by miRNAs, including miR-218, miR-181a and miR-227 $(23,25,26)$. To the best of our knowledge, the current study revealed that LEF1 is directly inhibited by miR- 6852 for the first time, and the overexpression of LEF1 reversed the impaired proliferation, migration and invasion of A172 cells induced by miR- 6852 .

In conclusion, the current study indicated that miR-6852 inhibited the proliferation, migration and invasion of glioma cells by inhibiting the expression of LEF1. Therefore, miR-6852 could be used as a novel biological target for the treatment of glioma. Subsequent data on how miR-6852 affects glioma should be obtained to provide a more reliable theoretical basis for this targeted glioma therapy.

\section{Acknowledgements}

Not applicable.

\section{Funding}

No funding was received.

\section{Availability of data and materials}

The datasets used and/or analyzed during the present study are available from the corresponding author on reasonable request.

\section{Authors' contributions}

JW and WD contributed to the conception and design of the present study. In addition, WD analyzed and interpreted the results and wrote the manuscript. JW, HL, KZ and SZ performed the experiments. All authors read and approved the final manuscript.

\section{Ethics approval and consent to participate}

The present study was approved by The Ethics Committee of Affiliated Hospital of Hebei University. Written informed consent was obtained from all enrolled patients.

\section{Patient consent for publication}

Not applicable.

\section{Competing interests}

The authors declare that they have no competing interests.

\section{References}

1. Lu B, Zhou Y, Su Z, Yan A and Ding P: Effect of CCL2 siRNA on proliferation and apoptosis in the U251 human glioma cell line. Mol Med Rep 16: 3387-3394, 2017.

2. Latha K, Yan J, Yang Y, Gressot LV, Kong L, Manyam G, Ezhilarasan R, Wang Q, Sulman EP, Xu J, et al: Abstract 5606: Fibrinogen-like protein 2 drives malignant tumor progression in glioma. Cancer Res 77: 5606, 2017.

3. Ellert-Miklaszewska A, Ciechomska IA and Kaminska B: Chapter e11 cannabinoid signaling in glioma cells and therapeutic implications. Handbook Cannabis Relat Pathologies: e111-e121, 2017.

4. Lv QL, Du H, Liu YL, Huang YT, Wang GH, Zhang X, Chen SH and Zhou HH: Low expression of microRNA-320b correlates with tumorigenesis and unfavorable prognosis in glioma. Oncol Rep 38: 959-966, 2017.

5. Kalogianni DP, Kalligosfyri PM, Kyriakou IK and Christopoulos TK: Advances in microRNA analysis. Anal Bioanal Chem 410: 695-713, 2018.

6. Santangelo A, Imbrucè P, Gardenghi B, Belli L, Agushi R, Tamanini A, Munari S, Bossi AM, Scambi I, Benati D, et al: A microRNA signature from serum exosomes of patients with glioma as complementary diagnostic biomarker. J Neurooncol 136: 51-62, 2018.

7. Zhang J, Lv J, Zhang F, Che H, Liao Q, Huang W, Li S and Li Y: MicroRNA-211 expression is down-regulated and associated with poor prognosis in human glioma. J Neurooncol 133: 553-559, 2017.

8. Li Y, Li Y, Ge P and Ma C: MiR-126 Regulates the ERK pathway via targeting KRAS to inhibit the glioma cell proliferation and invasion. Mol Neurobiol 54: 137-145, 2017.

9. Cui BH and Hong X: miR-6852 serves as a prognostic biomarker in colorectal cancer and inhibits tumor growth and metastasis by targeting TCF7. Exp Ther Med 16: 879-885, 2018.

10. Yu H, Zhang J, Wen Q, Dai Y, Zhang W, Li F and Li J: MicroRNA-6852 suppresses cell proliferation and invasion via targeting forkhead box J1 in gastric cancer. Exp Ther Med 16: 3249-3255, 2018.

11. Poudyal D, Herman A, Adelsberger JW, Yang J, Hu X, Chen Q, Bosche M, Sherman BT and Imamichi T: A novel microRNA, hsa-miR-6852 differentially regulated by Interleukin-27 induces necrosis in cervical cancer cells by downregulating the FoxM1 expression. Sci Rep 8: 900, 2018.

12. Livak KJ and Schmittgen TD: Analysis of relative gene expression data using real-time quantitative PCR and the 2(-Delta Delta C(T)) method. Methods 25: 402-408, 2001.

13. Lin L, Cai $\mathbf{J}$ and Jiang C: Recent advances in targeted therapy for glioma. Curr Med Chem 24: 1365-1381, 2017.

14. Jin C, Li M, Ouyang Y, Tan Z and Jiang Y: MiR-424 functions as a tumor suppressor in glioma cells and is down-regulated by DNA methylation. J Neurooncol 133: 247-255, 2017.

15. Xiao ZQ, Yin TK, Li YX, Zhang JH and Gu JJ: miR-130b regulates the proliferation, invasion and apoptosis of glioma cells via targeting of CYLD. Oncol Rep 38: 167-174, 2017.

16. Guo X, Zhang R, Liu J, Li M, Song C, Dovat S, Li J and Ge Z: Characterization of LEF1 high expression and novel mutations in adult acute lymphoblastic leukemia. PLoS One 10: e0125429, 2015.

17. Menter T, Trivedi P, Ahmad R, Flora R, Dirnhofer S, Tzankov A and Naresh KN: Diagnostic utility of lymphoid enhancer binding factor 1 immunohistochemistry in small B-cell lymphomas. Am J Clin Pathol 147: 292-300, 2017.

18. Santiago L, Daniels G, Wang D, Deng FM and Lee P: Wnt signaling pathway protein LEF1 in cancer, as a biomarker for prognosis and a target for treatment. Am J Cancer Res 7: 1389-1406, 2017.

19. Fang S, Lei LI, Liu M, Tsang J and Guan X: Abstract 2434: LEF1 negatively regulates differentiation of $\mathrm{HCC}$ by activation of Notch signaling pathway. Cancer Res 77: 2434, 2017.

20. Wang WJ, Yao Y, Jiang LL, Hu TH, Ma JQ, Ruan ZP, Tian T, Guo H, Wang SH and Nan KJ: Increased LEF1 expression and decreased Notch2 expression are strong predictors of poor outcomes in colorectal cancer patients. Dis Markers 35: 395-405, 2013. 
21. Gao X, Mi Y, Ma Y and Jin W: LEF1 regulates glioblastoma cell proliferation, migration, invasion, and cancer stem-like cell self-renewal. Tumor Biol 35: 11505-11511, 2014

22. Pećina-Šlaus N, Kafka A, Tomas D, Marković L, Okštajner PK, Sukser V and Krušlin B: Wnt signaling transcription factors TCF-1 and LEF-1 are upregulated in malignant astrocytic brain tumors. Histol Histopathol 29: 1557-1564, 2014.

23. Liang J, Li X, Li Y, Wei J, Daniels G, Zhong X, Wang J, Sfanos K, Melamed J, Zhao J and Lee P: LEF1 targeting EMT in prostate cancer invasion is mediated by miR-181a. Am J Cancer Res 5: 1124-1132, 2015.
24. Lyou Y, Habowski AN, Chen GT and Waterman ML: Inhibition of nuclear Wnt signaling: Challenges of an elusive target for cancer therapy. Br J Pharmacol 174: 4589-4599, 2017.

25. Qiu J, Hao Y, Huang S, Ma Y, Li X, Li D and Mao Y: MiR-557 works as a tumor suppressor in human lung cancers by negatively regulating LEF1 expression. Tumour Biol 39: 1010428317709467, 2017.

26. Liu Y, Yan W, Zhang W, Chen L, You G, Bao Z, Wang Y, Wang H, Kang $\mathrm{C}$ and Jiang T: MiR-218 reverses high invasiveness of glioblastoma cells by targeting the oncogenic transcription factor LEF1. Oncol Rep 28: 1013-1021, 2012. 